\title{
Sachregister zu Band 63
}

Die fett gedruckten Zahlen bezeichnen Eigenarbeiten. Bb. = Buchbesprechung.

Aalblutserum, Einfluß des - auf

das Auge 291. AbdominaleErkrankungen, $\mathrm{Pu}-$

pillenerscheinungen bei -384 . Ablatio retinae, eine seltene Form

der - im Innern eines sym-

pathisierenden Auges 201.

Entstehung der -370 .

Kolmotage bei der -314 .

macularis durch Blendung ? 48

Simulation einer Amaurose nach Spätheilung einer traumatischen - 388 .

Adap tierungszustand der Retina, Einfluß vegetativer Reiz-stoffe auf den - 299.

Aderhaut a. Chorioidea.

Adrenalin, Einwirkung des - bei Glaukom 122.

Adrenalinmydriasis, diagnosti-sche Verwertbarkeit der - 384.

- und Insulinmiosis 288.Akkommodationstonie 384.Amaurose, erhaltene Lichtreaktion der Pupille bei - 292.

- $\quad$ Simulation einer - - nach Spätheilung einer traumatischen Netz-hautablösung 388.

Amaurotische Idiotie 120. Amblyopie, Beseitigung der - bei

Schielenden 19. Anastomose, retinochorioideale -

der Arterie und Vene bei Con-

tusio bulbi 310. Anatomie des Auges 300 (Bericht). Aneurysma der Zentralgefäße, trau-

matisches - 203. Angioma orbitae 121. Angiomatosis retinae 118. Aniridia, Extractio cataractae bei

angeborener - 171, 308. Anisokorie bei Lungentuberkulose

384-Ankyloblepharon filiforme 321. Argya-Robertsonsches Phäno-

men 295, 296. Argyrosis corneae als Berufs-

krankheit 327. Astigmie, Zusammenhang zwischen

Conus myopicus und -312 . Atlas photographischer Bilder des

Augenhintergrundes 204 (Bb.) Augapfel s. Bulbus.

Auge, Schädigungendes - 329 (Bb.) Augenheilanstalten in Preußen

201. Augenhintergrund, Atlas photo-

graphischer Bilder des204

(Bb.)

- Lokalisation pathologischer \er-änderungen im - und ihre Projection auf die Außenseite derSklera 163.

Augenlider s. Lider. Augenoperationen, Bekämpf ung der Schmerzen nach - 117.

- Bulbusfixation bei den - 321.Augenstäbchen aus nichtrostendem Stahl 189. Augenverletzungen 309.

die an der Universitäts-Augen-klinik zu Königsberg behandelten - 71 . 
durch pflanzliche Gebilde 281.

typische Sportverletzungen beim Schilaufen 142.

B.

Besredka - Antivirus, Behand-

lung der Dacryocystitis mit -

308, 331. Bildende Kunst als Erkenntnis-

quelle für die Brillengeschichte

117. Bindehaut s. Conjunctiva. Binokularer Sehakt, Wiederher-

stellung des - bei Schielenden 19. Blattern-Schutzimpfung, Schä-

digung des Auges durch -- 338. Bleivergiftungen bei Üruckerei-

arbeitern, Nervensymptome bei

-379-

Blend ung, Ablatio retinae macularis durch - ? 48.

Blindenwesen 202, 203.

Bluter, Protrusis und Sehnerven-atrophie bei einem - 189 .

Blutungen retrobulbäre 312.

Brillengeschichte, die bildende Kunst als Erkenntnisquelle für die - 117 .

Bulbus, beiderseitige Spontanluxa-tion des - 188 .

Bulbusfixation bei den Augenoperationen 321.

Sachregister.

$4 \propto 3$

Bulbushyperämie, klinische Ver-

wendbarkeit der - 314-Buphthalmus 315.

C. Chlorylen 367. Chorioidea, Solitärtuberkulom der

- $\quad 3 ! 4 \cdot$ Chorioidealkarzinome, metasta-

tische 120, 186, 193. Chorioidealsarkome, Durchbruch von - 186.

- $\quad$ Stammbaum einer Familie mit

- $\quad$ 322.Chorioiditis 123.

- $\quad$ exsudativa unter dem Bilde derscheibenförmige Entartung derNetzhautmitte 205.

Chorioiditische Herde, periphere

$37^{\circ}$. Conjunctiva, primäre Tuberkulose

der-321. Contusiobulbi, retino-chorioideale

Anastomose der Arterie undVene

bei - 310. Conus myopicus, Zusammenhang

zwischen Astigmie und - 312. Cornea, experimentelle Studien

über die -184 .

Größe und Wachstum der - im kindlichen Alter 300.

perforierende Schnitt- und Riß-wunden der - 188.

umschriebene fettige Degeneration der - im Lidspaltenbezirk 188.

Corneanarben, operatives Verfah-

ren gegen - 304. Corneazysten 201. Corpus vitreum, ultramikroskopi-

sche Untersuchungen über die

Struktur des -300 .

1).

Dacryoadenitis acuta 308. Dacryocystitis, Behandlung der 
- $\quad$ mit Besredka-Antivirus 308,331.

Depigmentierung der Iris 316. Descemetsche Membran, Ablö-

sung der - durch Operation 316. Diabetes, Schwäche der glatten

Irismuskulatur bei - 292. a Druck, Entstehung des Augen-

druckes 299.

E.

Ebaga-Augensalben 307. Ektropium des Oberlides 304.

- uveae 394.

Elephantiasis des Oberlides, Zusammenhang der einseitigen - mit Erweiterung der Sella turcica $23 \delta$.

Elliotsche Operation, Katarakt nach - 197.

- $\quad$ Linsenextraktion nach - 314.Enophthalmus, Starextraktionbei

hochgradigem - 305. Entropium, Operation des spasti-schen - 304.

- $\quad$ Operationsverfahren gegen Tra-chom - des Oberlides 311.

Enzephalitis epidemica, Pupil -lenveränderungen bei - $377>378$, 379-

Epitheliom, mit Radium behan-deltes 114.

Ergotamin 290.

Exophthalmus, durch Wasser-stoffsuperoxyd verursachter - bei Tränensackexstirpation 305.

Exsudative Diathese und Tra-chom 313.

F.

Farbenlehre 330 (Bb.). Farbensehen, Theorie des - 320. Farbensinn der Fische 119. Fliegenlarve,

Entfernung einer le-

benden - aus der Vorderkammer

326. Flüssigkeitswechsel, intraoku-

larer 289, 299. Fremdkörper im Auge 118, 202.

- $\quad$ Herpes corneae nach Entfernungeines - 327.

Fremdkörperreflèx am Auge 299.

G. Geburt, toxische und mechanische

Ursachen der Sehstörung bei -

124. Getäßanomalien der Conjunctiva

und Sklera 192. Glaskörper s. Corpus vitreum. Glassplitter, Entfernung von -

aus der Vorderkammer 202. Glaukom, basale Iridektomie bei

inflammatorischem - 393-

- $\quad$ beiderseitige Irishypoplasie und

$-394$

Einwirkung des Adrenalin bei - 122 .

Glaukosanbehandlung des - 84, 308.

- Heterochromie - G. bei Mutter

und Tochter 113. Glaukosan 84, 308. G1 i a im Mittelhirn bei reflektorischer

Pupillenstarre 295. Grubenbildung auf der Papule 1 j 2

404 Sachregister.

$\mathrm{H}$.

Haenelsches Symptom 319. Hemiakinesis pupillaris 380. Hemianopsie, besondere Form der nasalen - 323. Herpes corneae nach Fremdkörper-

entfernung 327. Herpes zoster ophthalmicus.ein

Folgezustand des -292 . Herpes virus im Blut und Liquor 
242. Heterochromie - Glaukom bei

Mutter und Tochter 113. Histopathologie des Auges, Atlas

der - 204 (Bb.). Höllensteinverätzung 200. Hornhaut s. Cornea. Hydrocephalus, feinflockige

Pig-

mentdegeneration bei -394 . Hydrophthalmus und Feuermal

318. Hypertension, retinale Verände-

rungen bei arterieller -395 .

I.

Impfung, Schädigung des Auges

durch Blattern - Schutzimpfung

338. Infiltrationsanästhesie, sub-

konj unktivale bei Bulbusoperatio-

nen 248. Innervation, sympathische - des

Auges 317. Insulinmiosis und Adrenalinmy-

driasis 288. Iontophorese 192. Iridektomie, basale - bei inflam-

matorischem Glaukom und Hoch-

druck 393. Iris, angeborenes Fehlen des vorde-

ren Blattes der - 175 .

üepigmentierung der - 316. Irisatrophie, essentielle 399.

vererbte 115. Irishypoplasie, beiderseitige und

Glaukom 394. Irismuskulatur, Schwäche der

glatten - bei Diabetes 292. Iritis durch angeborene Syphilis

316.

- $\quad$ gonorrhoische 187.

K.

Kapillardruck, intraokularer 299. Karzinommetastasen in der Cho-

“ rioidea 120. Katarakt, endokrine Ätiologie des juvenilen - 197.

entzündungserregende Wirkung der Starreste 310.

nach Elliots Operation 197.

siderotische Naht -326 .

Katarakta senilis, Antagonismus frischer - und seniler Makula-degeneration 127, 400.

Spontanresorption der -102 .

Ursachen der und Kalziumgehalt des Blutes bei -303.

Kataraktoperationenander Uni-versitäts-Augenklinik Königsberg

bei angeborener Aniridia 171, 308.

bei hochgradigem Enophthalmus

305 .

Häufigkeit der senilen Makula-degeneration nach - 127, 400 .

retroiridische Jakobsonsche -- in.

Kautschuk, Überempfindlichkeit gegen - als Ursache von Urticaria und Çjuinckeschem Ödem 201.

Keratitisneuroparalytica durch Gewerbebeschädigung 201.

Keratitisparenchymatosa, Ent-stehung der - 324.

Keratoplastik, optische 197.

Klettenstachel, Verletzungen durch - 281.

Kolmotage bei der Netzhautab-lösung 314. 
Kolobom des gelben Fleckes 284.

Kristalle im Auge 119.

L.

Lebersche Krankheit 118. Lepra, klinische und histologische Befunde am leprösen Auge 254.

Lichtreflexe 296. Lidplastik 118. Lidrandplastik 302. Lidumkehrung, Instrument zur

Linsenextraktion im elliottrepa-

nierten Auge 314. Linsenluxation, angeborene 189.

- $\quad$ subkonjunktivale 305.Linsenschädigung durch Rönt-

genstrahlen 319. Linsentrübungen, medikamentö-se Behandlung der-192. I Lues 118.

- Differentialdiagnose zwischen Tu-berkulose und - des Sehorgans

$3 ! 5-$

- $\quad$ gleichzeitiges Vorkommen vonTuberkulose und - bei Augen-kranken 304.

- Scheintumoren der Orbita bei - 189. i Lues congenita, Iritis durch - 316 I Lungenbefund bei

Augentuberku-lose 198.

Sachregister. 405

Lungentuberkulose, Anisokorie

bei - 384. Luxatio bulbi durch Motorradfah-

rer 202.

M. Macula lutea, Anatomie der -300 .

Kolobom der -284 .

Nachweis der gelben Färbung der

- $\quad$ in gewöhnlichem Licht 192.Makuladegeneration, Antagonis-

mus zwischen seniler - und seni-ler Katarakt und Häufigkeit der

- $\quad$ nach Staroperation 127, 400.

- $\quad$ familiäre progressive 189.Magensaftsekretion und Pupil-

lenweite 289.

Melanosarkom der Iris 185.

Mercurochrom 312.

Microcoria familiaris 384.

Mikroskopische Untersuchun-gen des lebenden Auges mit star-ken Vergrößerungen 362.

Miosis congenita 384.

Mitbewegung, pathologische - der Pupille 383.

Molluscum contagiosum 321.

Myelitis, Pupillenerscheinungen bei -. 379.

Myopie 116. - Pupillenweite bei - 291.

Myosalvarsan 125.

N. Naevus flammeus und Hydroph-

thalmus 318. Natrium hydrocarbonicum 308. Nervus oculomotorius, Pupillen-

phänomen bei Lähmung des -

383. Nervus opticus, Erkrankung des

- durch Augenhöhleneiterung116.

- $\quad$ Atrophie, hereditäre 118.Nervus trigeminus, Einfluß des

- $\quad$ auf die Pupille des Kaninchens

- $\quad$ 382.Netzhaut s. Retina.Netzhautablösung s. Ablatio

retinae. Neurofibromatose des Auges 184,

$3^{\circ} 9,318$. Neurologie des Auges 288, 377 
(Berichte). Nystagmus, Pupillen-N. 383.

- latens 310 .

0.

Ö1 z y s t e , Trichloressigsäurebehand-

lung bei -394 . Ophthalmia electrica 198.

Ophthalmia sympathica 118 .

- $\quad$ Pigmentkomplementbindungsre-aktionen bei - 303.

Ophthalmologie 328 (Bb.). Opticus s. Nervus opticus. Orbitaeiterung, Sehnervenerkrankung durch -. 116. Orbitalphlegmone, doppelseitige

- $\quad$ nach stumpfem Schädeltraumabei einem Geisteskranken 200.

Orbitaltumoren, Piezometer zur Diagnose retrobulbärer - 136.

Scheintumoren bei Luetikern 189.

zwei - an einem Auge 309.

P.

Papule, Grubenbildung auf der

- 112.

Pemphigus conjunctivae 190, 192.

Pharynxreflex der Pupille 381.

Physiologie des Auges 298 (Be-richt).

Piezometer zur Diagnose retrobulbärer Orbitageschwülste 136.

Pigmentdegeneration, feinflok-kige - bei Hydrocephalus 394.

Pigmentepithel der Netzhaut, Rolle des - beim Sehakt 300.

Pigmentkomplementbindungs-reaktionen bei sympathischer Ophthalmie 303.

Psammom 65.

PseudoreflektorischePupillen-starre,Differentialdiagnose zwischen echter und - 293.

Ptosis, operierter Fall einer ange-borenen - 115.

Pupillenlehre 28811., 377ft., (Berichte) .

Pupillennystagmus 383.

Pupillenphänomene, wechselnde 223.

Pupillenstarre, Díf erentialdiag-nose zwischen echter und pseudo-reflektorischer - 293.

- $\quad$ Heilung einer durch Siderosisverursachten -309.

Pupillometer, optisches 291. Pupillotonie 384. Pyozyaneusinf ektion der Cornea

$327-$

.9-

Quinckesches Ödem, Überemp-findlichkeit gegen Kautschuk als Ursache von - 201.

R.

Radiotherapie, Internationale 126

(Bb.) Radium, mit - behandeltes Epi-

theliom 114.

406

Sachregister.

Raupenhaarverletzung des Auges 201. Ref lektorische Pupillenstarre

293 (Bb.) Retina, Chorioiditis exsudativa unter dem Bilde der scheibenartigen 
Entartung, der - mitte 205.

Einfluß vegetativer Reizstoffe auf den Adaptierungszustand der - 299.

funktionelle Uberlegenheit der na-salen Hälften der - 319 .

mitte, ein eigenartiger Fall von Veränderung der - 48.

Pupillenweite bei Pigmentdege-neration der - 291.

Veränderungen der - bei arteri-eller Hypertension 395.

Retinitis exsudativa 117.

- $\quad$ senile makularexsudative - 397-Retinocytom 319.Retinosis 123.

Rivanol 307. Röntgenstrahlen, Linsenschädi-

gung durch -319 . Röntgentherapie 306.

S. Schädeldachfrakturen, para-

doxe Pupillenreaktion infolge

von -379 . Schädeltrauma, doppelseitige Or-

bitalphlegmone nach stumpfem

- bei eínem Geisteskranken 200. Scheintumoren der Orbita 18gíí. Schilaufen, typische

Sportverlet-

zungen des Auges beim - 142. Schmerzen, Bekämpfung der

nach Augenoperationen 117. - und Pupille 380. Schwangerschaf t, toxische und

mechanische Ursachen der Seh-

störung bei - 124. Schwefelwasserbadeanlagen,

Augenschädigungen bei den Ar-

beitern der - 327. Schwimmbadkonjunktivitis,

Beziehungen der- zumTrachom

150. Sehnerv s. Nervus opticus. Sehschärfe, monokuläre und bino-

kuläre 159. Sella turcica, Zusammenhang

der einseitigen Elephantiasis des

Oberlides mit Erweiterung der - 235. Septoj odschädigung des Auges

Siderosis, Heilung einer durch -

verursachten Pupillenstarre 309.

Siderotische Nahtkatarakt 326.

Siebbeinzelleneiterung, sponta-ne Zurückbildung von Augener-scheinungen, die durch - verursacht wurden 113.

Sinus cavernosus, Thrombose des

- 308.Sportverletzungen, typische des

Auges beim Schilaufen 142. Sterilisation, trockene - der

Augeninstrumente 325. Strabismus, Beseitigung der Am-

blyopie und die Wiederherstellung

des binokularen Sehakts bei -19 .

- divergens, operierter Fall von an-geborenem - 115 .

Symblepharon congenitum 321. Sympathische In nervation des

Auges 317. Synchisis scintillans 321.

T.

Tarsomarginoplastik 302. Tetanie, Pupillenstörungen bei -

380. Thalliumvergiftung, gewerbliche

326. Trachom, Behandlung des -301 .

- $\quad$ Beziehungen der Schwimmbad-conjunctivitis zum - 150. 
- $\quad$ und exsudative Diathese 313.Trachomentropium des Oberlides

Operationsverfahren gegen - 311 Tränensackexstirpation, durch

Wasserstoffsuperoxyd verursach-

ter Exophthalmus bei $3^{\circ} 5$.

Trichloräthylen 807. Trichloressigsäurebehandlung

bei Ölzyste 394. Trypaflavin, intravenöse Anwen-

dung des -177 . Tuberkulorn, solitäres - der Cho-

rioidea 314. Tuberkulose des Auges 329 (Bb.)

- $\quad$ Differentialdiagnose zwischen Lues und - des Sehorgans 315.

- $\quad$ gleichzeitiges Vorkommen von Lues und - bei Augenkranken 304.

- $\quad$ Lungenbefund bei - - des Auges198.

- $\quad$ primäre - der Conjunctiva 321.Tumoren des hinteren Augenab-

schnittes, Diagnose der -184 .

- epibulbäre 310.

U. U1 cus serpens 118. Ultraviolettabsorption von Hornhaut und Linse, Messung der

- $\quad$ am lebenden Auge 38.Unfallkunde 199, 326, 388.Unf all neurose 199, 200.

Sachregister.

407

Untersuchungslampe 306. Urticaria, Überempfindlichkeit ge-

gen Kautschuk als Ursache von

-201 .

V.

Yakzinegeschwüre an den Lidern

118. Velonoskiaskopie 343. Vergiftungen, Merkblätter über

berufliche -199 .

Versicherungskunde 199, 326, 388.

Vivocoll 305.

W.

Wasserstoffsuperoxyd als Ursache eines Exophthalmus bei Tränensackexstirpation 305.

Z.

Zilien, Einheilung von - in die Hornhaut und Vorderkammer 326

Namenregister zu Banð 63.

A. Adlersberg 289. Albrich 304. Altherthum 384. Amsler 380. Archangelsky 327. Avizonis 235.

Awguschewitsch 331.

B.

Baeumler 192. Balod 201. Bar 292. Barath 384. Baurmann 299, 300. Becker, J., (Budapest)

US-Becker (Naumburg)

187. Beckershaus 188. Behr 293. Benders 117, 120. Berner 384. Betlheim 383. Bielschowsky

378. Birsch-Hirschfeld 1. Birnbacher 298. v. Blaskovics 301, 302,

303. 305. 311-Bliedung 291. Bonhoeffer 199. Breiger 388. Brons 198.

C. Car 292. Cholina 321. Christiansen 323. Clausen 185, 186, 191. Colrat 329. Comberg 194,

195. Cords 377. Cramer 184. v. Csapody 114, 115,

116. Curschmann 200.

D. 
Danilewsky 321. Davenport 322, 397. Deusch 379. Deutschmann 401 (P.) Dieter 317, 320.

Dimmer 204. Ditroi 305. Döhring 112. Dusseldorp 400.

E. E.Ikes 102. Enroth 197. Erggelet 184, 191. Erlanger 194, 196.

F.

Feilchenfeld 194. Fejér 113, 114, 305, 308 Feldmann 379. Ferenczy 311. Finkelnburg 381. Finn off 325. Fischer, F. P. 184. Fodor 150. Fraymann 124. Frieberg 197. Fuchs, A., 204.

G.

Gajewitsch 321. Georgieff 293. Ginsberg 195. Glüh 175. Görlitz 314. Grädatz 201. Gredsted 84. Griscom 399. Grönholm 197. v. Grósz 114, 309, 312,

. $3 * 3-$

Grünstein 293. Grüter 223, 330 (P.) Guist 394. Gutmann, A. 136. Gyotoku 254.

H.

Hamann 319. Hanke 329. Hanssen 319, 370. Hartmann 326. Hauptvogel 383. Heesch 300.

Heilbrun 203. Heine 300. Heintz 319. v. Herrenschwand

142. Hess 290, 299. van der Hoeve 120,

127, 400. vom Hofe 188. Hoffmann, W. 38. Hofmann, Edm. 125. Hollo s 306. Holth 384 Horay 113, 304. Horvath 306. Hudovernig 378.

I.

Igersheimer 201.

v. Imre jun. 114, 115,

$304.3^{\circ} 9 \cdot$ v. Imre sen. 113, 302,

310, 311, 313. Isakowitz 195.

J.

v. Jarmersted 71. Jese 338. jilik 313-

K.

Käding ..384. Kaiser 300

318. Kalinowsky 367. Kalk 384. Kassner 378. Kauders 289. Kestenbaum 159. Klauber 327. 